8 December, 2003

\title{
A new $\gamma^{*} p / \bar{p} p$ factorization test in diffraction, valid below $Q^{2} \sim 6 \mathrm{GeV}^{2}$
}

\author{
Samim Erhan and Peter Schlein \\ University of California*, Los Angeles, California 90095, USA.
}

\begin{abstract}
One of the key experimental issues in high energy hadron physics is the extent to which data from the diffractive interaction mechanism may be described by a factorized formula which is the product of a universal term describing the probability of finding a $\mathcal{P}$ omeron in a proton (loosely referred to as the "Pomeron flux-factor") and a term describing the Pomeron's interaction with the other incident proton. In the present paper, after demonstrating that existing data on diffractive $\gamma^{*} p$ and $\bar{p} p$ interactions show that the $\mathcal{P}$ omeron flux-factor is not universal, we present the results of a new test of factorization in these interactions which does not rely on universality of the flux-factor. The test is satisfied to within $\sim 20 \%$ for $1<Q^{2}<6 \mathrm{GeV}^{2}$ and $\beta \leq 0.2$ in the $\gamma^{*} p$ interactions, suggesting that the reasons for non-universality of the flux-factor have a limited effect on the factorization itself. However, a clear breakdown of this test is observed at larger $Q^{2}$. Kharzeev and Levin suggest that this can be attributed to the onset of QCD evolution effects in the $\mathcal{P}$ omeron's structure. The breakdown occurs in a $Q^{2}$ region which agrees with their estimates of a small $\mathcal{P}$ omeron size.
\end{abstract}

submitted to European Physical Journal C

* Supported by U.S. National Science Foundation Grant PHY-9986703 



\section{Introduction}

Studies of the inclusive inelastic production of beam-like particles with momenta within a few percent of the associated incident beam momentum, as in:

$$
\begin{aligned}
& \bar{p}+p_{i} \rightarrow X+p_{f} \\
& \gamma^{*}+p_{i} \rightarrow X+p_{f}
\end{aligned}
$$

have led to the development of a Regge phenomenology $[1,2]$ of these processes (see Fig. 1). The observed final-state proton momentum reflects the exchanged Pomeron's momentum fraction in the proton ${ }^{1}, \xi \equiv x_{\mathbb{P}}=1-x_{p}$, and exchanged momentum transfer squared, $t$.
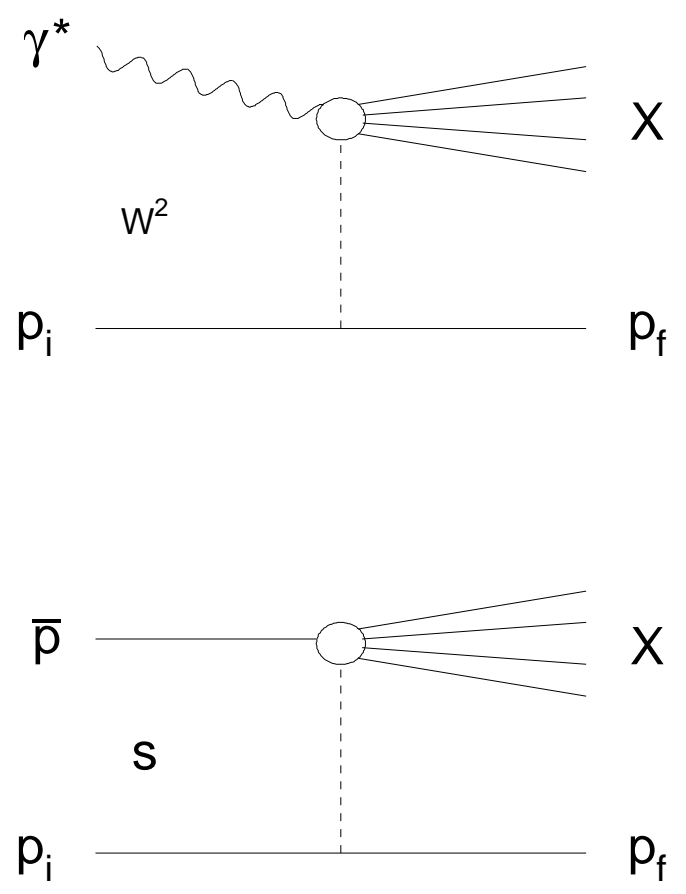

Figure 1: Upper: The diffractive $\gamma^{*}$-proton process. The total squared energy in the interaction is $W^{2}$. The 4-vector length squared of the $\gamma^{*}$ is $-Q^{2}$; Lower: The diffractive $\bar{p} p$ process. In each process, the exchanged $\mathcal{P}$ omeron has a squared 4 -momentum transfer, $t$, and momentum fraction, $\xi \equiv x_{\mathbb{P}}=1-x_{p}$, of the incident proton.

One of the relatively recent ideas [3] underlying the phenomenology is that, although the $\mathcal{P}$ omeron's existence in the proton is due to non-perturbative QCD, once the $\mathcal{P}$ omeron exists, perturbative QCD processes can occur in the proton-P $\mathcal{P}$ omeron and $\gamma^{*}$-P $\mathcal{P}$ omeron

${ }^{1}$ We use the symbol $\xi$ for this variable in view of its simplicity and its increasing use in the literature. 
interactions of Reacts. 1 and 2, respectively. Ref. [3] proposed the study of such hard processes in Reacts. 1 and 2 in order to determine the $\mathcal{P}$ omeron's structure. Hard diffraction scattering was discovered in $\bar{p} p$ interactions by the UA8 experiment [4] at the $\mathrm{S} p \bar{p} \mathrm{~S}-$ Collider and in ep interactions by the ZEUS [5] and H1 [6] experiments at HERA.

All available inclusive diffractive data from Reacts. 1 and 2 are well described [7, 8, 9] by expressing the observed single-diffraction differential cross sections as products of factors describing the $\mathcal{P}$ omeron flux in the proton, $F_{\mathcal{P} / p}(t, \xi)$ (hereafter referred to loosely but conveniently as $\mathcal{P}$ omeron emission), and $\mathcal{P}$ omeron interaction, for example proton$\mathcal{P}$ omeron or $\gamma^{*}-\mathcal{P}$ omeron total cross sections, respectively.

$$
\begin{gathered}
\frac{d^{2} \sigma_{\bar{p} p}^{\text {diff }}}{d \xi d t}=F_{\mathcal{P} / p}^{\bar{p} p}(t, \xi) \cdot \sigma_{p \mathcal{P}}^{\text {tot }}\left(s^{\prime}\right) \\
\frac{d^{2} \sigma_{\gamma^{*} p}^{\text {diff }}}{d \xi d t}=F_{\mathcal{P} / p}^{e p}(t, \xi) \cdot \sigma_{\gamma^{*} \mathcal{P}}^{\text {tot }}\left(s^{\prime}, Q^{2}\right)
\end{gathered}
$$

$-Q^{2}$ is the squared momentum transfer of the $\gamma^{*}$ in React. 2. $s^{\prime}$ is the squared invariant mass of the $X$ systems in Reacts. 1 and 2. To good approximation ${ }^{2}, s^{\prime}=\xi s$ in React. 1 and $s^{\prime}=\xi W^{2}-Q^{2}$ in React. 2 (see Fig. 1).

There is, however, one complicating issue in the successful description of the data by Eqs. 3 and 4 . The empirical Pomeron flux factors, $F_{\mathcal{P} / p}^{\bar{p} p}(t, \xi)$ and $F_{\mathcal{P} / p}^{e p}(t, \xi)$, in the two equations are found to be different. More specifically, the effective $\mathcal{P}$ omeron Regge trajectory in the common factor, $F_{\mathcal{P} / p}(t, \xi) \sim \xi^{1-2 \alpha(t)}$, required to fit the data is different in React. 1 and 2. The ZEUS [8] and H1 [9] collaborations both demonstrated that the effective $\mathcal{P}$ omeron trajectory at low- $|t|$ in React. 2 lies above the effective trajectory which characterizes React. 1 (the evidence for this is shown below in Sect. 3). This is a remarkable situation and tells us that, although all existing data are well described by Eqs. 3 and 4, the Pomeron flux factor in the proton is not universal.

This conclusion should not come as a surprise because, for example, Kaidalov et al. [10] predicted that higher-order non-perturbative $\mathcal{P}$ omeron-exchange effects in $p p$ interactions lead to an effective $\mathcal{P}$ omeron Regge trajectory whose intercept at $t=0$ decreases with increasing energy. Moreover, as summarized in the following section, we have reported [11] such effects ${ }^{3}$ by fitting Eq. 3 to all available data on React. 1. Presumably, similar but weaker effects should also take place in ep colisions.

In the present paper, despite the non-universality of the $\mathcal{P}$ omeron flux factor in Reacts. 1 and 2, we propose to test the factorization represented in Eqs. 3 and 4. We accomplish this by asking if the $\mathcal{P}$ omeron-exchange components of the extracted $\gamma^{*}$-P omeron and $p$-Pomeron total cross sections satisfy the relationship:

$$
\frac{\sigma_{\gamma^{*} \mathcal{P}}^{\mathrm{tot}}}{\sigma_{\gamma^{*} p}^{\mathrm{tot}}}=\frac{\sigma_{p \mathcal{P}}^{\mathrm{tot}}}{\sigma_{p p}^{\mathrm{tot}}},
$$

\footnotetext{
${ }^{2}$ The second equation comes from $s^{\prime}+Q^{2}-t=\xi\left(W^{2}+Q^{2}\right)$, when $|t|<<s^{\prime}$ and $\xi<<1$.

${ }^{3}$ Only below $s \sim 550 \mathrm{GeV}^{2}$ is the effective $\mathcal{P}$ omeron trajectory equal to the trajectory which describes the $s$-dependence of the $p p$ and $p \bar{p}$ total cross sections $[12,13]$.
} 
where the denominators are the total $\gamma^{*} p$ and $p p$ cross sections, respectively, and where all four are evaluated at the same cms interaction energy [14]. Equation 5 is obtained from the optical theorem and the ratios of the forward elastic amplitudes shown in Fig. 2.

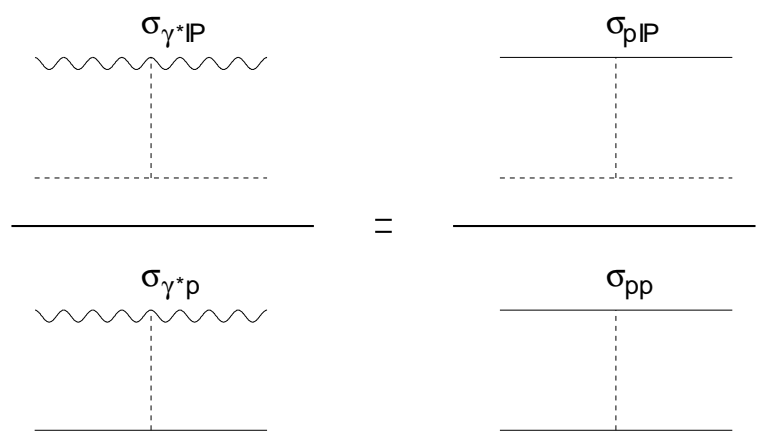

Figure 2: Ratios of the $\gamma^{*}$ and hadronic forward elastic amplitudes referred to in the text. In all cases, the dashed lines are Pomerons, the solid lines are protons and the curved lines are $\gamma^{*} s$. On both left and right sides, the upper vertices cancel, showing that each is the ratio of $\mathcal{P}$ omeron-P $\mathcal{P}$ omeron to $\mathcal{P}$ omeron-proton vertices. Hence, the left and right sides should be equal.

However, Eq. 5 can not be used directly. In extracting the $\gamma^{*}$-P omeron and $p$-P Pomeron cross sections from the data using Eqs. 3 and 4, in each case only the product of a flux factor normalization constant, $K$, and the cross section is experimentally accessible. However, since $F_{\mathcal{P} / p}(t, \xi)$ is not universal, $K$ may also not be universal. Thus, we introduce the notation, $K_{e p}$ and $K_{p p}$ for the two cases, respectively, and the test of Eq. 5 is actually a test of:

$$
\frac{K_{e p} \sigma_{\gamma^{*} \mathcal{P}}^{\text {tot }}}{\sigma_{\gamma^{*} p}^{\text {tot }}}=\frac{K_{p p} \sigma_{p \mathcal{P}}^{\text {tot }}}{\sigma_{p p}^{\text {tot }}},
$$

If Eq. 6 is found to agree with data, as seems to be the case (see Sects. 4 and 5), the simplest explanation is that $K_{e p} \approx K_{p p}$ and the extracted cross sections obey Eq. 5 .

In Sect. 2 we review the existing phenomenological analyses of React. 1 in terms of Eq. 3 and extract the right-hand side of Eq. 6. In Sect. 3 the HERA diffractive data on React. 2 is reanalyzed and the left-hand side of Eq. 6 is extracted from the data. In Sect. 4, the factorization test is carried out using Eq. 6. Our conclusions are given in Sect. 5 .

\section{Review of diffractive $\bar{p} p$ and $p p$ data analysis}

The UA8 collaboration [7] fit Eq. 3 to the joint $\xi$ - $t$ distributions of the available data on React. 1 at the $\operatorname{Sp} p \mathrm{~S}\left(\sqrt{s}=630 \mathrm{GeV}\right.$ and $\left.1.0<|t|<2.0 \mathrm{GeV}^{2}\right)$ and the corresponding $p p$ data at the ISR $[15]\left(\sqrt{s}=23.5,30.5 \mathrm{GeV}\right.$ and $\left.|t|<2.0 \mathrm{GeV}^{2}\right)$, all with $0.03<\xi<0.09$. 
They obtained parametrizations of $F_{\mathcal{P} / p}(t, \xi)$ and $\sigma_{p \mathcal{P}}^{\text {tot }}$ which embody some features not previously known:

$$
\begin{gathered}
F_{\mathcal{P} / p}(t, \xi)=K_{p p} \cdot\left|F_{1}(t)\right|^{2} \cdot e^{(1.1 \pm 0.2) t} \cdot \xi^{1-2 \alpha(t)} \\
\alpha(t)=1+\epsilon+\alpha^{\prime} t+\alpha^{\prime \prime} t^{2}=1.10+0.25 t+(0.079 \pm 0.012) t^{2} \\
K_{p p} \sigma_{p \mathcal{P}}^{\text {tot }}\left(s^{\prime}\right)=(0.72 \pm 0.10) \cdot\left[\left(s^{\prime}\right)^{0.10}+(4.0 \pm 0.6)\left(s^{\prime}\right)^{-0.32}\right] \mathrm{mb} \mathrm{GeV}^{-2} .
\end{gathered}
$$

With $\left|F_{1}(t)\right|^{2}$ in Eq. 7 set equal to the Donnachie-Landshoff [16] form factor ${ }^{4}$, the additional exponential factor is required.

The fits show that the effective $\mathcal{P}$ omeron Regge trajectory flattens in the domain, $1.0<|t|<2.0 \mathrm{GeV}^{2}$, as described by by the quadratic term in Eq. 8, when $\epsilon$ and $\alpha^{\prime}$ are fixed at 0.10 and $0.25 \mathrm{GeV}^{-2}$, respectively. In Eq. 9, with the exponents ${ }^{5}$ of $s^{\prime}=\xi s$ fixed at 0.10 and -0.32 , respectively, $K_{p p} \sigma_{p \mathcal{P}}^{\text {tot }}\left(s^{\prime}\right)$ requires the presence of both $\mathcal{P}$ omeronand Reggeon-exchange terms, as shown. Ref. [7] confirms the flattening of the effective trajectory at larger $|t|$ values, as well as the presence of the Reggeon-exchange term in Eq. 9, by fitting the observed $\xi$-dependences at fixed $t$ values when ${ }^{6} \xi<0.03$. In the fits, the experimental resolution and geometrical acceptance are taken into account.

A description of the phenomenology of React. 1 is incomplete without inclusion of the explicit effects of multi-P omeron-exchange. It has been widely known for some time that the observed $s$-dependence of the total single-diffractive cross section, $\sigma_{\bar{p} p}^{\text {tot diff }}$, is not described by Eq. 3 (integrated over $t$ and $\xi<0.05$ ) with a fixed $\mathcal{P}$ omeron Regge trajectory. Such a calculated $\sigma_{\bar{p} p}^{\text {tot diff }}$ rises rapidly with energy and soon violates unitarity, while the observed $\sigma_{\bar{p} p}^{\text {totdiff }}$ tends to level off or plateau at high energy $[17,18]$. Since there is no built-in mechanism in the single-Pomeron-exchange process of Fig. 1 to account for the plateauing of $\sigma_{\bar{p} p}^{\text {tot diff }}$, there have been continuing theoretical efforts to satisfy $s$-channel unitarity [19]; this effect is attributed to multiple-Pomeron-exchange and is referred to variously in the literature as damping, screening, shadowing or absorption. Kaidalov et al. [10] showed that multi-P Pomeron-exchange diagrams lead to an effective $\mathcal{P}$ omeron trajectory whose $t=0$ intercept decreases with increasing energy.

In order to assess these effects quantitatively, Ref. [11] performed fits of Eq. 3 integrated over $\xi<0.05$ to the $d \sigma / d t$ of all available ISR [20] and $\operatorname{S} \bar{p} p \mathrm{~S}[7,21]$ data. In fitting to the complete set of ISR $d \sigma / d t$ data over the energy range, $s=549$ to $3840 \mathrm{GeV}^{2}$, the only free parameters in Eqs. 7, 8 and 9 were those in the effective Pomeron trajectory, each of which was assumed to have a simple $s$-dependence. The fit results from Ref. [11] $\operatorname{are}^{7}$ :

\footnotetext{
${ }^{4} F_{1}(t)=\frac{4 m_{p}^{2}-2.8 t}{4 m_{p}^{2}-t} \cdot \frac{1}{(1-t / 0.71)^{2}}$

${ }^{5}$ In this formula and others like it, " $s$ " stands for " $s$ ' $/ s_{0}$ ", where $s_{0}=1 \mathrm{GeV}^{2}$.

${ }^{6}$ The sensitivity of fitting at small $\xi$ comes from the fact that, at small momentum transfer, the rapid increase of $\xi^{1-2 \alpha(0)} \sim 1 / \xi^{1+2 \epsilon}$ dominates the relatively weak dependence of $\sigma_{p \mathcal{P}}^{\text {tot }}$ on $\xi$ (via $s^{\prime}=\xi s$ ).

${ }^{7}$ The logarithms are to base 10 .
} 


$$
\begin{aligned}
& \epsilon(s)=(0.096 \pm 0.004)-(0.019 \pm 0.005) \cdot \log (s / 549) \\
& \alpha^{\prime}(s)=(0.215 \pm 0.011)-(0.031 \pm 0.012) \cdot \log (s / 549) \\
& \alpha^{\prime \prime}(s)=(0.064 \pm 0.006)-(0.010 \pm 0.006) \cdot \log (s / 549)
\end{aligned}
$$

At the lowest ISR energy, $s=549 \mathrm{GeV}^{2}, \epsilon=0.096, \alpha^{\prime}=0.215 \mathrm{GeV}^{-2}$ and $\alpha^{\prime \prime}=$ $0.064 \mathrm{GeV}^{-4}$, while each of these is seen to decrease with increasing energy. This is consistent with fixing $\epsilon=0.10$ and $\alpha^{\prime}=0.25$ in the fits of Ref. [7], since the only low- $|t|$ data in those fits were at the lowest ISR energies.

Ref. [11] finds that the effective $\mathcal{P}$ omeron trajectory continues to decrease at higher energy. At the $\mathrm{S} \bar{p} p \mathrm{~S},(\sqrt{s}=630 \mathrm{GeV})$, the effective trajectory is:

$$
\alpha(t)=1+\epsilon+\alpha^{\prime} t+\alpha^{\prime \prime} t^{2}=1.035+0.165 t+0.059 t^{2}
$$

Ref. [11] also shows that this $\alpha(t)$ form is consistent with the published function [22] that is said to describe the CDF data on React. 1 at the Tevatron.

For completeness, we note that the fits of Ref. [7] are in kinematic regions where multi$\mathcal{P}$ omeron-exchange effects in React. 1 seem to be smallest. In Refs. [11, 18] it is shown that the effective $\alpha(t)$ is relatively independent of $s$ at the low end of the ISR energy range and that there is no evidence for $s$-dependence of the effective $\alpha(t)$ in the $|t|-$ range, $1-2$ $\mathrm{GeV}^{2}$ (its average value is $0.92 \pm 0.03$.). Thus, multi- $\mathcal{P}$ omeron-exchange effects appear to be mainly in the low- $\xi$, low- $|t|$ region $[11,18]$, where most of the cross section is.

To prepare for the factorization test of Eq. 6, we need to evaluate its right-hand-side. Its numerator is given by Eq. 9, while its denominator is the $p p$ total cross section, which we take from the fits of Refs. [12, 13]:

$$
\sigma_{p p}^{\text {tot }}=18 s^{0.10}-27 s^{-0.50}+55 s^{-0.32} \mathrm{mb} .
$$

Since we are interested only in the $\mathcal{P}$ omeron exchange terms in Eqs. 9 and 10, we drop the Reggeon-exchange terms in both numerator and denominator. The right-hand-side of Eq. 6 is then given by:

$$
\frac{K_{p p} \sigma_{p \mathcal{P}}^{\text {tot }}}{\sigma_{p p}^{\text {tot }}}=0.041 \pm 0.007 \mathrm{GeV}^{-2}
$$

\section{Analysis of diffractive $\gamma^{*} p$ data}

In order to carry out the factorization tests, we first reanalyze HERA $e p$ diffractive data samples. The diffractive structure function, $\xi F_{2}^{D(3)}$, for the ZEUS 1994 data [8] is displayed in Fig. 3 and for the H1 1994 data [9] in Fig. 4. The errors shown are obtained by summing the squared statistical and systematic errors, respectively. In both experiments, the recoil proton was not detected and the data are therefore integrated over $t$. This also means that the proton recoil system includes a low-mass excitation component, which was measured at the CERN ISR [23] to be $(12.0 \pm 2.5) \%$ of the recoil system. This leads 


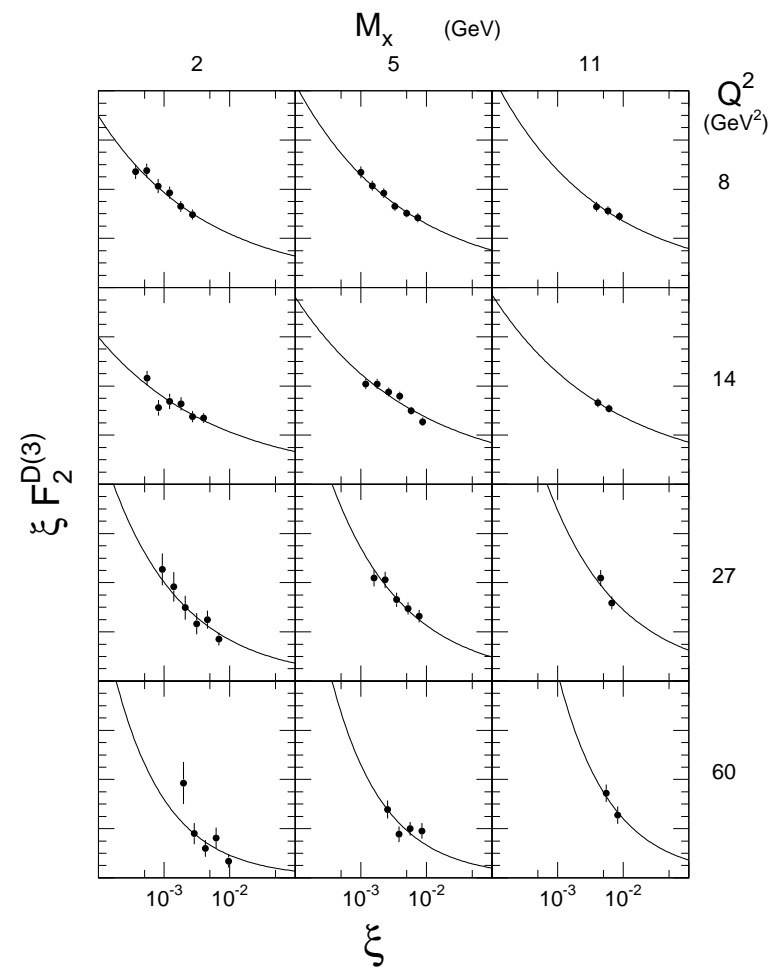

Figure 3: The ZEUS 1994 data [8]: $\xi F_{2}^{D(3)}$, vs. $\xi\left(\xi \equiv x_{\mathbb{P}}\right)$ for 12 sets of $\left(M_{X}, Q^{2}\right)$ values. At fixed $M_{X}$ and $Q^{2}$, $\xi$ and $W^{2}$ are uniquely related $\left(\xi=\left(M_{X}^{2}+Q^{2}\right) / W^{2}\right)$. Thus, each set of points displays the $W^{2}$ dependence of React. 2 at fixed $M_{X}$ and $Q^{2}$. The curves are the results of fitting Eq. 14 to the points shown, as discussed in the text.

to a small systematic upward shift in the $F_{2}^{D(3)}$ points, which is corrected for in our final Fig. 7.

Following standard usage $[24,8,9]$, the diffractive structure function, $F_{2}^{D(3)}$, is related to the diffractive $\gamma^{*}$-proton differential cross section by ${ }^{8}$ :

$$
\frac{d \sigma_{\gamma^{*} p}^{\text {diff }}}{d \xi}=\frac{4 \pi^{2} \alpha}{Q^{2}} \cdot F_{2}^{D(3)}\left(\beta, Q^{2}, \xi\right)
$$

where, as noted earlier, the symbol, $\xi \equiv x_{\mathbb{P}}$, is used.

As in Ref. [3], $F_{2}^{D(3)}$ is written in factorized form, as the product of a $\mathcal{P}$ omeron flux factor (in this case, integrated over $t$ ) and a $\mathcal{P}$ omeron structure function, $F_{2}^{D(2)}$ :

$$
F_{2}^{D(3)}\left(\beta, Q^{2}, \xi\right)=\int F_{\mathcal{P} / p}^{e p}(t, \xi) d t \cdot F_{2}^{D(2)}\left(\beta, Q^{2}\right) \approx \frac{K_{e p}}{\xi^{1+2 \epsilon} \cdot\left(3.9-2 \alpha^{\prime} \ln \xi\right)} \cdot F_{2}^{D(2)}\left(\beta, Q^{2}\right) .
$$

\footnotetext{
${ }^{8}$ Eq. 12 is obtained from Eq. 8 of Ref. [8].
} 
or:

$$
\xi F_{2}^{D(3)}\left(\beta, Q^{2}, \xi\right)=\frac{K_{e p} F_{2}^{D(2)}\left(\beta, Q^{2}\right)}{\xi^{2 \epsilon} \cdot\left(3.9-2 \alpha^{\prime} \ln \xi\right)} .
$$

This approximate form of the flux factor integrated over $t$ arises from assuming $e^{3.9 t} \xi^{1-2 \alpha(t)}$ for the functional form of $F_{\mathcal{P} / p}(t, \xi) .3 .9$ is the value which makes the integral equal to that of the $|t|$-integral of the full flux factor in Eq. 7 when $\alpha^{\prime}=0.25$ is used ${ }^{9}$.

Fig. 3 shows the fits of Eq. 14 to the ZEUS data. The free parameters are $\epsilon$ and an independent $K F_{2}^{D(2)}$ at each of the twelve $Q^{2}$ and $M_{X}$ combinations ( $\alpha^{\prime}$ is fixed at +0.25 $\mathrm{GeV}^{-2}$ ). These fits, and those made to the $\mathrm{H} 1$ data in Fig. 4, confirm factorization of $\mathcal{P}$ omeron production and interaction in the diffractive $\gamma^{*} p$ interactions. In the domain, $\xi<0.01$, where Reggeon exchange can be ignored at all $\beta$, all the observed dependence on $\xi$ is described by the flux factor in Eq. 14. Although this factorization has been known for some time from the $\mathrm{H} 1$ and ZEUS experiments, it is perhaps not widely recognized how remarkable it is.

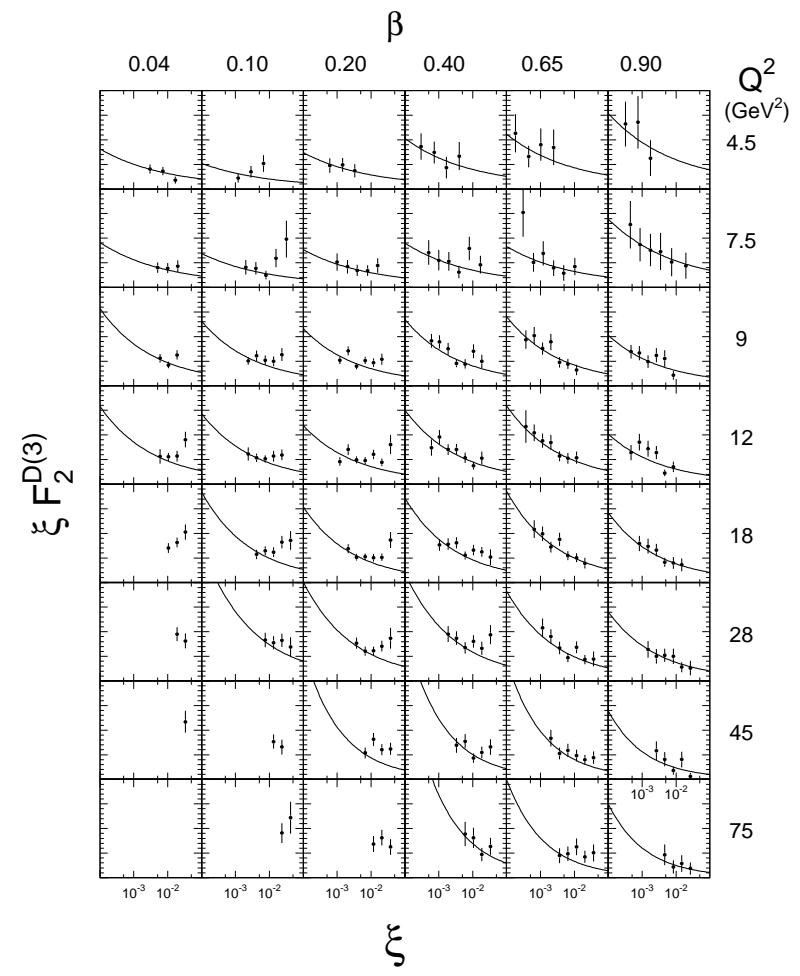

Figure 4: The H1 1994 data [9]: $\xi F_{2}^{D(3)}$, vs. $\xi\left(\xi \equiv x_{\mathbb{P}}\right)$ in bins of $\beta$ and $Q^{2}$. At each $Q^{2}, s^{\prime}=Q^{2} \cdot(1-\beta) / \beta$. The curves are the results of fitting Eq. 14 to the points with $\xi<10^{-2}$, as discussed in the text.

\footnotetext{
${ }^{9}$ This constant decreases to 3.7 and 3.5 , for $\alpha^{\prime}=0.15$ and 0.05 , respectively.
} 
From the fits to the ZEUS data, Fig. 5 shows the results when we fix $\alpha^{\prime}$ at the series of four values shown and determine $\epsilon$ and the twelve normalization constants in Fig. 3. Fig. 5 shows a $1 \sigma$ error "band" of allowed $\alpha^{\prime}$ and $\epsilon$ values. All points along the valley of the contour are equally acceptable as solutions and there is insignificant discrimination between them with the present data. Although we assume $\alpha^{\prime}=+0.25$ for the factorization analysis in this paper, we note that if the true effective $\alpha^{\prime}$ were as small as +0.15 , the final ratios used in the factorization analysis only change by about $10 \%$ and do not effect our conclusions.

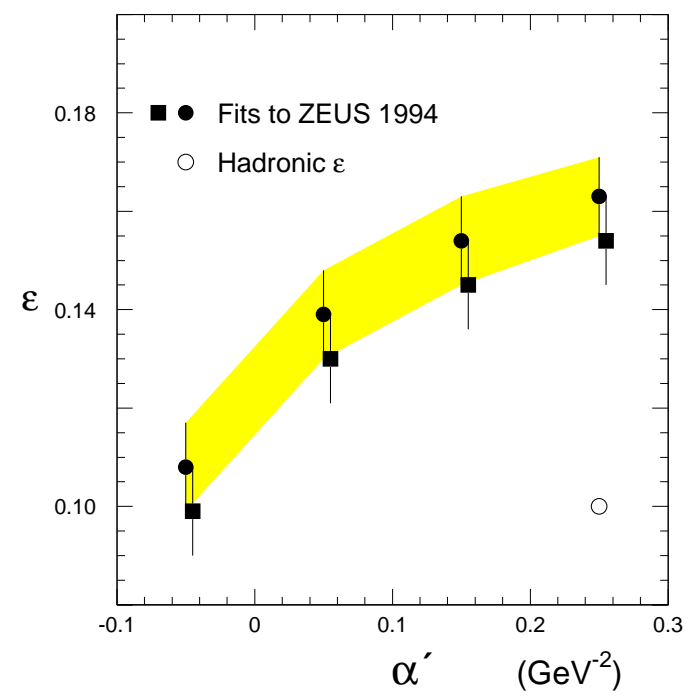

Figure 5: Fitted $\epsilon$ vs. fixed $\alpha^{\prime}$ from fits to the ZEUS 1994 data shown in Fig. 3. The solid circles are from fits to all four $Q^{2}$ ZEUS data sets; the solid squares are from fits to only the two lowest $Q^{2}$ data sets. The shaded band represents the $\pm \sigma$ fit contour in the first set. The open circle shows the "soft" Pomeron trajectory parameters, obtained from fitting the $s$-dependence of total $p p$ and $\bar{p} p$ cross sections.

We note in Fig. 5 that the band of allowed $\epsilon$ and $\alpha^{\prime}$ values for the $\gamma^{*}-\mathrm{p}$ interactions is seen to be inconsistent with the conventional hadronic "soft- $\mathcal{P}$ omeron" effective Regge trajectory parameters $[12,13,16], \epsilon=0.10$ and $\alpha^{\prime}=+0.25$. This had been noted earlier by both ZEUS [8] and H1 [9], but only for the assumed value, $\alpha^{\prime}=+0.25$. We note here that the disagreement holds no matter what value of $\alpha^{\prime}$ is assumed.

Fig. 6 shows the fitted values of $\epsilon$ vs. $Q^{2}$ for fixed $\alpha^{\prime}=+0.25$. The ZEUS points correspond to simultaneous fits to the three distributions at each $Q^{2}$ shown in Fig. 3. The H1 points are from combined fits made to the distributions with $\xi<10^{-2}$, at each two neighboring $Q^{2}$ values (4.5 and 7.5, 9 and 12, etc.) shown in Fig. 4. There is a suggestion that $\epsilon$ depends on $Q^{2}$. 


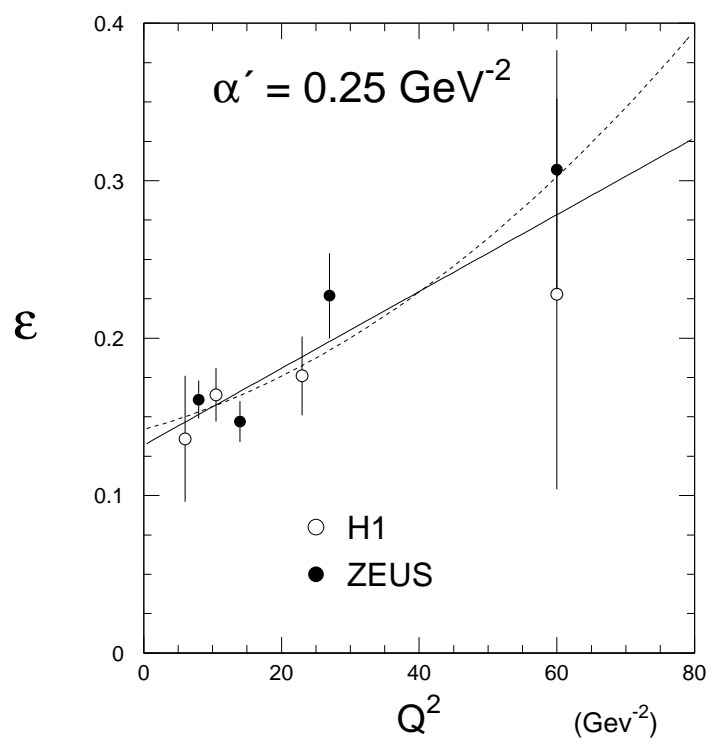

Figure 6: Fitted values of $\epsilon$ vs. $Q^{2}$ with $\alpha^{\prime}$ fixed at $0.25 \mathrm{GeV}^{-2}$, as explained in the text. The solid and dashed curves are, respectively, linear and quadratic fits to the points shown.

\section{Factorization test}

To express the numerator on the left-hand-side of Eq. 6 in terms of the measured structure function, a comparison of Eqs. 12 and 13 with Eq. 4 yields:

$$
K_{e p} \sigma_{\gamma^{*} \mathcal{P}}^{\mathrm{tot}}\left(s^{\prime}, Q^{2}\right)=\frac{4 \pi^{2} \alpha}{Q^{2}} \cdot K_{e p} F_{2}^{D(2)}\left(\beta, Q^{2}\right)
$$

where:

$$
s^{\prime}=Q^{2}(1-\beta) / \beta
$$

The denominator on the left-hand-side of Eq. $6, \sigma_{\gamma^{*} p}^{\text {tot }}$, is approximately given in terms of the $F_{2}$ structure function by:

$$
\sigma_{\gamma^{*} p}^{\text {tot }}\left(W^{2}, Q^{2}\right)=\frac{4 \pi^{2} \alpha}{Q^{2}} \cdot F_{2}\left(x, Q^{2}\right)
$$

where, because both $\sigma_{\gamma^{*} \mathcal{P}}^{\text {tot }}$ and $\sigma_{\gamma^{*} p}^{\text {tot }}$ are evaluated at the same CM energy [14], $W^{2}=s^{\prime}$ and $x=Q^{2} /\left(W^{2}+Q^{2}-m_{p}^{2}\right)$. Our desired ratio is:

$$
\text { Ratio } \equiv \frac{K_{e p} \sigma_{\gamma^{*} \mathcal{P}}^{\text {tot }}}{\sigma_{\gamma^{*} p}^{\text {tot }}}
$$

Fig. 7 shows the Ratio defined in Eq. 18 evaluated vs. $Q^{2}$ for three different $\mathrm{H} 1$ data samples at $\beta=0.04,0.10,0.20$ and 0.40 . For all points, the numberator is obtained 


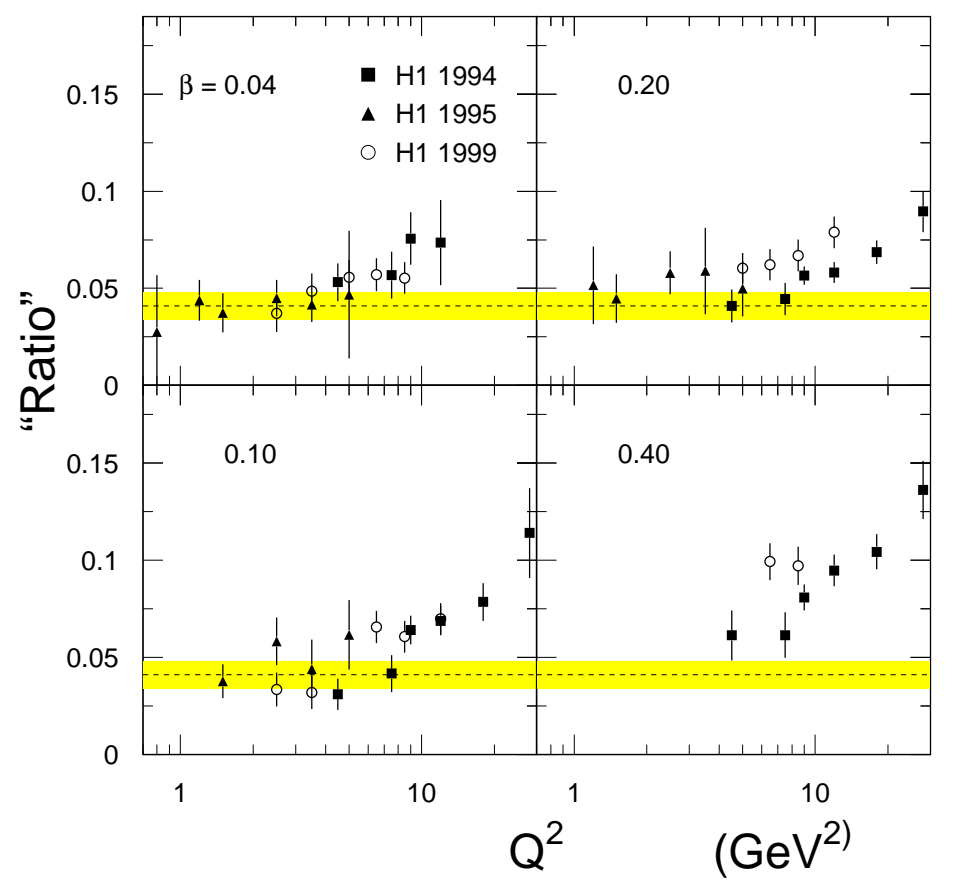

Figure 7: Ratio defined in Eq. 18 vs. $Q^{2}$ for $\beta=0.04,0.10,0.20$ and 0.4. The solidsquare points are calculated using the $1994 \mathrm{H1}$ data [9] and are the ratios of $K_{e p} \sigma_{\gamma^{*} \mathcal{P}}^{\text {tot }}$ to $\sigma_{\gamma^{*} p}^{\text {tot }}$ as explained in the text. The solid triangles and open circle points use the preliminary lower-Q ${ }^{2} 1995$ [26] and 1999 [27] H1 data as measured from figures in their conference papers. The shaded band is the ratio of $K_{p p} \sigma_{p \mathcal{P}}^{\text {tot }}$ to $\sigma_{p p}^{\text {tot }}$; see Eq. 11. In the extraction of the $K_{e p} \sigma_{\gamma^{*} \mathcal{P}}^{\text {tot }}$ values, $\epsilon=0.15$ and $\alpha^{\prime}=0.25$ are used. All points are systematically shifted downward by 12\% to account for excitation of the unobserved proton, as discussed in Sect. 3.

from Eq. 15 and the denominator, $\sigma_{\gamma^{*} p}^{\text {tot }}$, was calculated using the parameterization of Abramowicz and Levy [25].

The solid-square points in Fig. 7 with $4.5<Q^{2}<28 \mathrm{GeV}^{2}$ are calculated using the $1994 \mathrm{H} 1$ data [9]. We see in the figure that these points are in reasonable agreement with the factorization prediction in Eq. 11 at the lower end of their $Q^{2}$ range (4.5 and $7.5 \mathrm{GeV}^{2}$ ) and $\beta<0.4$, while for $Q^{2}$ larger than 6 or $7 \mathrm{GeV}^{2}$, there is a clear divergence from agreement with the prediction. The points plotted in Fig. 7 with solid triangles at lower $Q^{2}$ values, $0.8<Q^{2}<5 \mathrm{GeV}^{2}$, are preliminary results from the $1995 \mathrm{H} 1$ data [26]. Those plotted with open circles in the range, $2.5<Q^{2}<12 \mathrm{GeV}^{2}$, are preliminary results from the $1999 \mathrm{H} 1$ data [27]. The points are seen to be in reasonable agreement in the $Q^{2}$ domains where they overlap. Eq. 6 , seems to be satisfied to within $20 \%$ below $Q^{2} \sim 6$ or $7 \mathrm{GeV}^{2}$ and $\beta<0.4$.

As discussed in the following section, the breakdown of Factorization for $Q^{2}$ above about $6 \mathrm{GeV}^{2}$ can be attributed to the onset of perturbative QCD efects on a small 
Pomeron. This is agreement with the magnitude calculated by Kharzeev and Levin [28]. However, several caveats concerning the results in Fig. 7 should be noted.

1. The factorization prediction of Eq. 6 is only valid for its $\mathcal{P}$ omeron-exchange components. Although we have these for the right-hand-side of Eq. 6, as shown in Eq. 11 and the bands in Fig. 7, we are presently unable to know the $\mathcal{P}$ omeron-exchange components in the left-hand-side of Eq. 6, or the data points in Fig. 7. Thus, agreement between bands and data points in Fig. 7 implies that $\mathcal{P}$ omeron-exchange is the same fraction of both numerator and denominator of Ratio.

2. In calculating the points in Fig. $7, \epsilon=0.15$ and $\alpha^{\prime}=0.25$ are assumed. It is relevant to point out that the character of Fig. 7 is not very sensitive to uncertainties in these parameters. For example, as noted in the previous section, if $\alpha^{\prime}=+0.15$ is used to calculate the ratios in the figure, their values change by only $\sim 10 \%$ and the conclusions do not change.

3. We pointed out above that the $K$ factor in $F_{\mathcal{P} / p}(t, \xi)$ might be different in Reacts. 1 and 2 and we therefore labeled them differently. However, the approximate factorization agreement that we find at the lower $Q^{2}$ values implies that the two $K$ values are probably not very different.

\section{Conclusions}

We have summarized the phenomenology of inclusive single diffraction in $p p(\bar{p} p)$ interactions in which all available data are well described by a product of two functions which describe, respectively, the $\mathcal{P}$ omeron flux in a proton and the $\mathcal{P}$ omeron-proton cross section. The $\mathcal{P}$ omeron flux factor has the characteristic Regge form, except that the empirical $\mathcal{P}$ omeron Regge trajectory is an effective one whose $t=0$ intercept and slope decrease with increasing energy. Following the arguments of Kaidalov et al. [10], this presumably reflects multi- $\mathcal{P}$ omeron-exchange processes which grow with energy, although it seems surprising that the factorized formula continues to describe the data as well as it does under these circumstances.

The data on diffractive $\gamma^{*} p$ interactions in HERA ep collisions are also well described by a product of a Pomeron flux factor and a cross section factor. However, the effec-

tive $\mathcal{P}$ omeron trajectory is distinctly different from what is found in the corresponding hadronic interactions referred to in the previous paragraph. A possible interpretation for this fact is that multi- $\mathcal{P}$ omeron-exchange effects are different in $p p$ and $e p$ collisions.

From fits to the two diffractive data sets, we have extracted values for the $\gamma^{*}-\mathcal{P}$ omeron and $p$-P omeron total cross sections (in each case multiplied by the normalization constant of the respective $\mathcal{P}$ omeron flux factor). We then combined these cross sections with the known total $\gamma^{*} p$ and $p p$ total cross sections to test a simple factorization relation between their $\mathcal{P}$ omeron exchange components due to the optical theorem. 
The factorization test is observed to be reasonably well satisfied, to within about $20 \%$, in the range, $1<Q^{2}<6 \mathrm{GeV}^{2}$. However, at higher $Q^{2}$ values, a clear breakdown in the factorization test is observed.

In view of the pronounced and different $\mathcal{P}$ omeron trajectory intercepts which are observed in the two classes of reactions, the first of these two observations is very surprising. It seems to be telling us that the dominant multi-Pomeron-exchange (or damping) effects are of such a nature that factorization of the Pomeron's flux factor and its interaction is not very much disturbed.

According to Kharzeev and Levin [28], our second observation concerning the breakdown of factorization observed at larger $Q^{2}$ can be understood in terms of the onset of QCD evolution effects in the $\mathcal{P}$ omeron structure and a small $\mathcal{P}$ omeron size (see also Ref. [29]). Arguing that the properties of the soft $\mathcal{P}$ omeron are linked to the scale anomaly of QCD, Kharzeev and Levin calculate that the scale, $M_{0}^{2} \sim 4 \div 6 \mathrm{GeV}^{2}$ is the largest non-perturbative scale in QCD. This corresponds to $Q^{2} \sim 1 / R_{\mathcal{P}}^{2} \sim M_{0}^{2}$, where $R_{\mathcal{P}}$ is a typical size of the $\mathcal{P}$ omeron.

The $Q^{2}$ value at which our observed breakdown occurs gives a measure of the size of the Pomeron: $R_{\mathcal{P}}^{2} \sim 1 / Q^{2}=\left(0.39 \mathrm{GeV}^{2} \mathrm{mb}\right) /\left(6 \mathrm{GeV}^{2}\right)=0.065 \mathrm{mb}$. The area, $\pi R_{\mathcal{P}}^{2}=0.20 \mathrm{mb}$, agrees well with the recent UA8 measurement [30] of the $\mathcal{P}$ omeron$\mathcal{P}$ omeron total cross section, $\sigma_{\mathcal{P} \mathcal{P}}^{\text {tot }}=0.2 \mathrm{mb}$ above a center of mass energy of about 10 $\mathrm{GeV}$. In this connection, it is also interesting to note that UA8 also obtained a statistically modest, but significant, test of factorization in double-P $\mathcal{P}$ omeron-exchange:

$$
\bar{p} p \rightarrow \bar{p} X p
$$

using the relation:

$$
\frac{K^{2} \sigma_{\mathcal{P} \mathcal{P}}^{\text {tot }}}{K \sigma_{p \mathcal{P}}^{t o t}}=\frac{K \sigma_{p \mathcal{P}}^{\text {tot }}}{\sigma_{p p}^{\text {tot }}} .
$$

As pointed out in Sect. 4, there are limitations to the factorization analysis presented in this paper. These can be addressed by obtaining larger and improved event samples. For example, in the case of React. 2, more detailed $t$-dependent measurements will allow an unambiguous determination of the effective $\mathcal{P}$ omeron trajectory, espectially as a function of $Q^{2}$. The left-hand side in Eq. 6 should be determined for the $\mathcal{P}$ omeron-exchange component alone. In the case of React. 1 , there is a great need for new and more complete data samples over a wider range of $t$ and $s$. This will be necessary in order to understand how the validity of our factorization test depends on the degree of damping in the reactions. This may be a very important issue, which we were not able to address in this paper because of a lack of the necessary data. To pursue this topic in the future, it will be necessary to have detailed studies of React. 1 at the Tevatron and a possible new experiment at RHIC, which can cover the energy range between ISR and S $\bar{p} p \mathrm{~S}$.

In summary, we conclude that, despite the non-universality of the $\mathcal{P}$ omeron flux factor in Reacts. 1 and 2, the differential cross sections for these reactions can, to good approximation, still be written as a product of $\mathcal{P}$ omeron formation and interaction factors. In other words, there is no evidence for a breakdown of the factorization embodied in Eqs. 3 
and 4 for $Q^{2}<6 \mathrm{GeV}^{2}$ in React. 2. The apparent factorization breakdowns reported in Refs. [31, 32, 33] are likely due to the different effective $\mathcal{P}$ omeron trajectories in ep and pp interactions.

\section{Acknowledgements}

We thank John Dainton and Guenter Wolf for extensive, invaluable discussions about diffractive $\gamma^{*} p$ interactions and providing us with the 1994 H1 and ZEUS diffractive data sets. We are greatly indebted to Dima Kharzeev for pointing out the significance of our results in terms of his work with Genya Levin. We also thank Alexei Kaidalov, Boris Kopeliovich, Uri Maor and Misha Ryskin for helpful discussions. We are grateful to CERN, where most of this work was done, for their continued hospitality. 


\section{References}

[1] see: P.D.B. Collins, "An Introduction to Regge Theory \& High Energy Physics", Cambridge University Press (1977), and references therein.

[2] See, for example:

A.H. Mueller, Phys. Rev. D 2 (1970) 2963; D 4 (1971) 150;

A.B. Kaidalov et al., Pisma JETP 17 (1973) 626;

A. Capella, Phys. Rev. D 8 (1973) 2047;

R.D. Field and G.C. Fox, Nucl. Phys. B 80 (1974) 367;

D.P. Roy and R.G. Roberts, Nucl. Phys. B 77 (1974) 240;

A.B. Kaidalov, Phys. Reports 50 (1979) 157.

[3] G. Ingelman and P.E. Schlein, Phys. Lett. B 152 (1985) 256.

[4] R. Bonino et al. (UA8 Collaboration), Phys. Lett. B 211 (1988) 239;

A. Brandt et al. (UA8 Collaboration), Phys. Lett. B 297 (1992) 417;

A. Brandt et al. (UA8 Collaboration), Phys. Lett. B 421 (1998) 395.

[5] M. Derrick et al. (ZEUS Collaboration), Phys. Lett. B 315 (1993) 481.

[6] T. Ahmed et al. (H1 Collaboration), Nucl. Phys. B429 (1994) 477.

[7] A. Brandt et al. (UA8 Collaboration), Nucl. Phys. B 514 (1998) 3.

[8] J. Breitweg et al. (ZEUS Collaboration), Europ. Phys. Jour. C 6 (1999) 43 and references therein.

[9] C. Adloff et al. (H1 Collaboration), Zeit. f. Physik C 76 (1997) 613.

[10] A.B. Kaidalov, L.A. Ponomarov, K.A. Ter-Martirosyan, Sov. Jour. Nucl. Phys. 44 (1986) 468.

[11] S. Erhan and P. Schlein, Phys. Lett. B 481 (2000) 177.

[12] J.R. Cudell, K. Kang and S.K. Kim, Phys. Lett. B 395 (1997) 311; see also: "Simple Model for Total Cross Sections", preprint, Brown-HET-1060, January 1997.

[13] R.J.M. Covolan, J. Montanha \& K. Goulianos, Phys. Lett. B 389 (1996) 176.

[14] M. Ryskin, private communication (1998).

[15] M.G. Albrow et al., Nucl. Phys. B54 (1973) 6;

M.G. Albrow et al., Nucl. Phys. B72 (1974) 376.

[16] A. Donnachie \& P.V. Landshoff, Nucl. Phys. B 231 (1984) 189; Nucl. Phys. B267 (1986) 690; Nucl. Phys. B 244 (1984) 322.

[17] K. Goulianos, Phys. Lett. B 358 (1995) 379; B 363 (1995) 268. 
[18] S. Erhan and P. Schlein, Phys. Lett. B 427 (1998) 389; B 445 (1999) 455.

[19] See, for example:

A. Capella, J. Kaplan and J. Tran Thanh Van, Nucl. Phys. B 105 (1976) 333;

V.A. Abramovsky, R.G. Betman, Sov. Jour. Nucl. Phys. 49 (1989) 747;

P. Aurenche et al., Phys. Rev. D 45 (1992) 92;

E. Gotsman, E.M. Levin and U. Maor, Zeit. Phys. C 57 (1993) 667; Phys. Rev. D 49 (1994) R4321; Phys. Lett B 353 (1995) 526.

[20] M.G. Albrow et al., Nucl. Phys. B 108 (1976) 1;

J.C.M. Armitage et al., Nucl. Phys. B 194 (1982) 365.

[21] M. Bozzo et al. (UA4 Collaboration), Phys. Lett. B 136 (1984) 217;

D. Bernard et al., Phys. Lett. B 186 (1987) 227.

[22] F. Abe et al. (CDF Collaboration), Phys. Rev. 50 (1994) 5535.

[23] L. Baksay et al. (R603 Collaboration) Phys. Lett. B 55 (1975) 491.

[24] G. Ingelman and K. Prytz, Zeit. f. Physik C 58 (1993) 285; Proc. Workshop "Physics at HERA", ed. W. Buchmueller and G. Ingelman, DESY 1992, Vol. 1, 233.

[25] H. Abramowicz and A. Levy, "The ALLM parametrization of $\sigma_{t o t}\left(\gamma^{*} p\right)$; an update", DESY 97-251, hep-ph/9712415 (and private communication from the authors: There are misprints in Table 2 ; the quoted values of $b_{\mathcal{P} 1}, b_{\mathcal{P} 2}, b_{\mathcal{R} 1}, b_{\mathcal{R} 2}$ should be squared. In Eq. 3, both occurrences of $Q_{o}^{2}$ should be replaced by $Q_{o}^{2}+\Lambda^{2}$.

[26] H1 Collaboration, "Measurements of the diffractive structure function, $F_{2}^{D(3)}$, at low and high $\mathrm{Q}^{2}$ at HERA", submitted to the 29th International Conference on HighEnergy Physics ICHEP98, Vancouver, Canada (July 1998) Abstract 571. The $\xi F_{2}^{D(3)}$ values we use at $\xi=0.005$ were obtained from their analog representions in Fig. 4.

[27] H1 Collaboration, "Measurement of the Diffractive Deep- Inelastic Scattering Cross Section at Low $Q^{2}$ " submitted to the 31st International Conference on High-Energy Physics ICHEP02, Amsterdam (July 2002) Abstract 981. The $\xi F_{2}^{D(3)}$ values we use at $\xi=0.005$ were obtained from their analog representions in Fig. 6 .

[28] D. Kharzeev and E. Levin, Nucl. Phys. B578 (2000) 351, hep- ph/9912216, and private communications.

[29] B.Z. Kopeliovich, "From hard to soft diffraction and return", hep-ph/0012104.

[30] A. Brandt et al. (UA8 Collaboration), Eur. Phys. Jour. C 25 (2002) 361.

[31] L. Alvero, J.C. Collins, J. Terron and J.J. Whitmore, Phys. Rev. D59 (1999) 074022.

[32] T. Affolder et al. (CDF Collaboration), Phys. Rev. Lett. 84 (2000) 5043.

[33] R.J.M Covolan and M.S. Soares, Phys. Rev. D 60 (1999) 054005. 